\title{
Prenatal risk factors and neonatal DNA methylation in very preterm infants
}

\author{
Marie Camerota ${ }^{1,2^{*}}$ (D, Stefan Graw ${ }^{3}$, Todd M. Everson ${ }^{3,4}$, Elisabeth C. McGowan ${ }^{5}$, Julie A. Hofheimer 6 , \\ T. Michael $\mathrm{O}^{\prime}$ Shea ${ }^{6}$, Brian S. Carter ${ }^{7}$, Jennifer B. Helderman ${ }^{8}$, Jennifer Check ${ }^{8}$, Charles R. Neal ${ }^{9}$, \\ Steven L. Pastyrnak ${ }^{10}$, Lynne M. Smith ${ }^{11}$, Lynne M. Dansereau² ${ }^{2}$ Sheri A. DellaGrotta ${ }^{2}$, Carmen J. Marsit ${ }^{3}$ and \\ Barry M. Lester ${ }^{1,2,5}$
}

\begin{abstract}
Background: Prenatal risk factors are related to poor health and developmental outcomes for infants, potentially via epigenetic mechanisms. We tested associations between person-centered prenatal risk profiles, cumulative prenatal risk models, and epigenome-wide DNA methylation (DNAm) in very preterm neonates.

Methods: We studied 542 infants from a multi-center study of infants born $<30$ weeks postmenstrual age. We assessed 24 prenatal risk factors via maternal report and medical record review. Latent class analysis was used to define prenatal risk profiles. DNAm was quantified from neonatal buccal cells using the Illumina MethylationEPIC Beadarray.

Results: We identified three latent profiles of women: a group with few risk factors (61\%) and groups with elevated physical (26\%) and psychological (13\%) risk factors. Neonates born to women in higher risk subgroups had differential DNAm at $2 \mathrm{CpG}$ sites. Higher cumulative prenatal risk was associated with methylation at $15 \mathrm{CpG}$ sites, 12 of which were located in genes previously linked to physical and mental health and neurodevelopment.

Conclusion: We observed associations between prenatal risk factors and DNAm in very preterm infants using both person-centered and cumulative risk approaches. Epigenetics offers a potential biological indicator of prenatal risk exposure.
\end{abstract}

Keywords: Prenatal, Methylation, Epigenetics, Epigenome-wide association study (EWAS), Neonatal, Preterm, Buccal

\section{Background}

Infants born less than 30 weeks postmenstrual age (PMA) are at increased risk for adverse health and developmental outcomes. As children, they are at high risk for experiencing chronic health problems related to brain injury, including cerebral palsy, autism spectrum disorder, seizures, epilepsy, mental health disorders [1-4] and developmental delay in motor, language, and cognitive

*Correspondence: marie_camerota@brown.edu

1 Department of Psychiatry and Human Behavior, Alpert Medical School of Brown University, Providence, RI, USA

Full list of author information is available at the end of the article domains [1-3, 5-7]. However, there is also marked heterogeneity in outcomes [8-10]. For example, a recent follow-up study from the Neonatal Research Network (NRN) cohort of infants born extremely preterm found that by age 2 , one quarter (24\%) of children had no neurodevelopmental impairment, and $45 \%$ had only suspected or mild impairment [7].

Adverse prenatal conditions contribute to risk of preterm birth, and may also exacerbate the risk of negative outcomes associated with immaturity and illness in very preterm children [11]. For example, maternal mood disorders (e.g., depression, anxiety) and medical complications (e.g., pre-eclampsia, pre-pregnancy obesity) during 
pregnancy predict poorer neurobehavioral outcomes in very preterm neonates [11], which in turn are associated with longer term impairments [12]. Sociodemographic risk factors, such as low socioeconomic status, are also associated with poor developmental outcomes for very preterm children [13]. While these adverse conditions arise from unique sources (physical, psychological, and sociodemographic), they may impact similar biological systems and could have additive effects on the developing fetus. Therefore, exposure to a greater number or specific combinations of risk factors in the prenatal environment may contribute to the heterogeneous outcomes observed among preterm children.

One mechanism by which prenatal conditions may alter child neurodevelopment is via epigenetic processes [14]. Epigenetics refers to molecular processes that regulate gene expression without altering the underlying DNA sequence. DNA methylation (DNAm) is the most commonly studied epigenetic mechanism in humans and involves addition of a methyl group to a cytosine-phosphate-guanine $(\mathrm{CpG})$ dinucleotide on a strand of DNA. DNAm plays an important role in regulating gene activity and expression. Additionally, offspring DNAm is sensitive to variations in environmental experience [15-17] and therefore may provide information about the biological embedding of prenatal conditions [14, 18-20].

Perhaps the most studied of all prenatal risk factors are those indicative of maternal psychological distress, including perceived stress and mood disorders [21]. These factors have also been studied in a growing number of candidate gene and epigenome-wide association studies (EWAS) [22, 23]. While candidate gene studies show associations between psychological risk factors in pregnancy and DNAm in genes implicated in offspring stress response systems [15], more recent EWAS on the same psychological risk factors have produced mixed findings [24-31], with differences not easily explained by study sample size. Therefore, the extent to which psychological risk factors in pregnancy impact offspring DNAm remains unclear. Physical risk factors (e.g., pre-pregnancy body mass index [BMI]) have also been investigated in relation to offspring DNAm and have similarly been associated with differential neonatal DNAm at a handful of CpGs [32]. These previous findings should be interpreted in light of several limitations, including the use of small sample sizes, exclusive use of cord blood for DNA sampling, and use of convenience or low-risk samples. Finally, most previous studies have investigated individual risk factors (e.g., depression, obesity) in isolation, rather than comprehensively assessing multiple facets of prenatal stress.

In this study, we investigated the relationship between prenatal risk factors and DNAm using buccal cell specimens in a high-risk population: children born very preterm. In addition, we studied prenatal risk comprehensively using two multiple-risk-factor approaches, rather than an individual variable approach. We first used cumulative risk models to investigate the additive burden of increasing number of risk factors on neonatal DNAm. Second, we used person-centered models to investigate the relationship of different types of risk factors with neonatal DNAm. Person-centered approaches such as latent class (LCA) and latent profile analysis (LPA) group individuals with similar co-occurring risk factors or phenotypes into mutually-exclusive groups. Whereas one previous study investigated cumulative prenatal risk in association with neonatal DNAm [24], person-centered models have not yet been used to study the association between prenatal risk phenotypes and neonatal DNAm. Therefore, the goals of this study were to examine relations among prenatal risk factors and DNAm in very preterm neonates and to understand whether these relations differ depending on whether cumulative risk or personcentered models are used. Addressing these goals may enable us to identify important biological mechanisms underlying the association between prenatal environmental experiences and child outcomes and will provide information regarding how best to operationalize prenatal risk factors in future studies of neonatal health.

\section{Methods \\ Study population}

The Neonatal Neurobehavior and Outcomes in Very Preterm Infants (NOVI) study enrolled infants born $<30$ weeks postmenstrual age (PMA) from nine NICUs affiliated with six universities from April 2014 to June 2016. Inclusion criteria included: (a) birth $<30$ weeks PMA; (b) parental ability to read and speak English or Spanish; and (c) residence within $3 \mathrm{~h}$ of the NICU and follow-up clinic. Infants were excluded for major congenital anomalies [33], NICU death, maternal age $<18$ years, maternal cognitive impairment, or maternal death.

Parents of eligible infants were invited to participate in the study when survival to discharge was determined to be likely by the attending neonatologist. Study procedures were explained and informed consent was obtained in accordance with each institution's review board. Children were included in this analysis if they were enrolled in NOVI at birth and had a neonatal buccal swab collected $\left(M_{\mathrm{PMA}}=39.2\right.$ weeks). There were 704 infants enrolled in NOVI; of these 651 (92\%) had parental consent to obtain buccal swabs. Mothers were interviewed at enrollment to obtain demographic information (age, education, occupation, race/ethnicity, and marital/ cohabitation status). Information regarding prenatal 
substance use, physical health, and psychological health were obtained via maternal interview and medical record review.

\section{Measures \\ Prenatal risk factors}

We assessed 24 prenatal risk factors in four domains: demographic (5 items), substance use (4 items), physical health (9 items), and psychological health (6 items). Individual risk factors were assessed via maternal interview and medical record review.

Demographic risk factors included maternal age $>35$ years, low socioeconomic status (Hollingshead category 5) [34], maternal education less than a high school degree, minority race or ethnicity, and no romantic partner. Substance use items included maternal use of tobacco, alcohol, marijuana, or other illegal substances (e.g., heroin, cocaine) as noted in her medical record.

Physical health risks included maternal underweight $(\mathrm{BMI}<18.5)$ and obesity $(\mathrm{BMI} \geq 30)$, calculated from reported pre-pregnancy height and weight. Gestational weight gain that exceeded Institute of Medicine guidelines [35] was also determined using calculated BMI and reported weight gain. Maternal hypertension, preeclampsia, diabetes, HIV/AIDS or other sexually transmitted infection, any other infection, and receipt of prenatal care were all determined from medical record review.

Psychological health risks included maternal depression and anxiety and maternal moods and feelings. Maternal depression during pregnancy was determined from medical record or maternal report of anti-depressant use, or from maternal report of depression diagnosis, treatment, or counseling during pregnancy. The same method was used to determine maternal anxiety during pregnancy. Beyond diagnosed mental health disorders, maternal moods and feelings during pregnancy were assessed from four questions asking mothers to indicate the extent to which (a) their pregnancy was a hard time in their lives, and the extent to which they felt (b) down, (c) hopeless, and (d) slow during their pregnancy. Risk was determined by responses indicating that pregnancy was a "very hard time" or "one of the worst times" in their lives, or if mothers indicated they "often" or "always" felt down, hopeless, or slow [36].

\section{Neonatal DNA methylation (DNAm)}

Genomic DNA was extracted from buccal swab samples, collected near term-equivalent age, using the Isohelix Buccal Swab system (Boca Scientific), quantified using the Quibit Fluorometer (Thermo Fisher, Waltham, MA, USA) and aliquoted into a standardized concentration for subsequent analyses. DNA samples were plated randomly across 96-well plates and provided to the Emory University Integrated Genomics Core for bisulfite modification using the EZ DNA Methylation Kit (Zymo Research, Irvine, $C A$ ), and subsequent assessment of genome-wide DNAm using the Illumina MethylationEPIC Beadarray (Illumina, San Diego, CA) following standardized methods based on the manufacturer's protocol.

Pre-processing of data followed a modified workflow described elsewhere [37]. Array data were normalized via Noob normalization [38, 39] and samples with more than $5 \%$ of probes yielding detection $p$-values $>1.0 \mathrm{E}-5$ or mismatch between reported and predicted sex were excluded. In addition, one of two duplicated samples was omitted (retained duplicated sample with smallest detection $p$-values). Probes with median detection $p$-values $<0.05$, probes measured on the $\mathrm{X}$ or $\mathrm{Y}$ chromosome, probes that had single nucleotide polymorphisms (SNP) within the binding region or that could cross-hybridize to other regions of the genome were excluded [40]. Then, array data were standardized across Type-I and Type-II probe designs with beta-mixture quantile normalization $[41,42]$. After exclusions, 706,323 probes were available from 542 samples for this study (83\% of 651 with buccal swab consent; $77 \%$ of entire NOVI cohort). These data are accessible through NCBI Gene Expression Omnibus (GEO) via accession series GSE128821.

\section{Covariates}

DNAm varies by cell type and cellular heterogeneity is a documented source of confounding in EWAS that make use of mixed cell samples [43]. A variety of cell-type deconvolution methods have been developed to estimate cell type proportions based on cell-type specific DNAm pattern. We estimated the proportion of epithelial, fibroblast, and immune cells (e.g., B-cells, natural killers, CD4+and CD8+T-cells, monocytes, neutrophils, eosinophils) in our buccal samples using reference methylomes [44]. As previously shown [45], for $95 \%$ of our buccal samples, $95.7 \%$ of the cells were epithelial cells, with the remainder being immune cells. Given the strong inverse correlation between epithelial and immune cell proportions, cellular heterogeneity was adjusted for by including the proportion of epithelial cells as a covariate in all statistical models.

In addition to cellular heterogeneity, our EWAS models controlled for child sex, recruitment site, and PMA at buccal swab. We accounted for potential batch effects by controlling for sample plate.

\section{Statistical analysis}

Prenatal risk classes and index

We first conducted latent class analysis (LCA) to categorize subgroups of women with similar prenatal risk 
factors. LCA is a statistical method for classifying individuals into mutually exclusive groups, or latent classes, based on their pattern of responses to a set of categorical indicator variables. The method is considered latent because true group membership is unknown. LCA employs maximum likelihood estimation. The optimal number of latent classes is determined using fit statistics and interpretibility of the models. For these analyses, we fit LCA models to our 24 observed risk factors and examined solutions ranging from 1 to 4 classes. All models were run in Mplus 8.4. We used posterior probabilities from the best fitting LCA model to classify women into distinct subgroups. We describe the subgroups in terms of how they differ on the 24 prenatal risk factors.

Next, we created a cumulative prenatal risk index that measured the total number of risk factors experienced by mothers. One point was assigned for each of 24 risk factors and a proportion score was created by dividing this sum by the total number of items mothers responded to. A proportion was used rather than a sum because of the possibility of item nonresponse. However, the majority of mothers $(96 \%)$ had data for all 24 items.

\section{Epigenome-wide association study (EWAS)}

All EWAS analyses were conducted in R 4.0.2. We used generalized estimating equations (GEE) that accounted for the nesting of children within families. GEE models were run on logit transformed $\left(\log \left(\frac{\beta}{1-\beta}\right)\right)$ DNAm data that approximated a Gaussian distribution. For easier interpretation of significant $\mathrm{CpG}$ sites, we present model coefficients obtained from both transformed and untransformed (beta-values) data, where the latter can be interpreted in terms of percent methylation at a given CpG site. To account for multiple testing, $p$-values were adjusted using a Bonferroni correction $(\alpha=0.05 / 706323)$. We conducted separate EWAS analyses with latent prenatal class (3-level factor) and cumulative prenatal risk (continuous) as the focal independent variables and compare our findings from the two types of models. We report results from models that yielded suggestive associations (FDR $<10 \%)$ in Additional file 1 and report those results that were significant with Bonferroni-correction (706,323 tests) herein.

One challenge of EWAS in humans is the inaccessibility of tissues of interest, namely brain tissue. Although we rely on peripheral tissues such as buccal cells, there is variability in the extent to which peripheral DNAm is associated with DNAm in the brain. For CpGs that were significantly associated with prenatal risk in either latent class or cumulative risk models, we examined the correlation between DNAm in buccal and brain tissue [46]. This additional information can help us determine which of our significant CpGs may have similar patterns of DNAm in buccal and brain tissue.

To determine the biological functions of $\mathrm{CpGs}$ associated with prenatal risk, we conducted gene enrichment analyses using the gometh function in the MissMethyl package [47]. This procedure accounts for the number of CpGs annotated to each gene. We examined both pathway-based gene sets (i.e., KEGG and gene ontology (GO) terms). For enrichment analyses, we included CpGs that were associated with prenatal risk at an FDR of $<5 \%$. Overrepresentation results within a $10 \%$ FDR were deemed statistically significant. We also aimed to identify whether any CpGs associated with prenatal risk were within genes that have been linked with neurodevelopmental phenotypes. Thus, based on the genes that were annotated to our significant CpGs, we additionally annotated these CpGs with traits that have been linked to these genes via prior genome-wide association studies (GWAS) using the NHGRI-EBI GWAS catalog [48].

All analyses described thus far have described methods for estimating the association between prenatal risk and individual CpGs. However, DNAm is generally highly correlated at adjacent CpG sites [49]. To better understand whether our EWAS results are limited to individually significant CpGs or are more broadly representative of regions of the genome that are differentially methylated, we additionally conducted differentially methylated regions (DMR) analysis using the $d m r f f$ package [50].

\section{Results \\ Study population}

The NOVI study included 704 infants born to 601 mothers. All mothers were included in the LCA analysis. Of the 651 potential buccal swabs, 624 (96\%) were collected. Missing data were due to technical sampling or handling error, missing swabs, or unscheduled discharges prior to swabs being obtained. Of the 624 infants with buccal swabs, there were 542 infants (from 470 mothers) with DNAm data that passed quality control steps (described earlier).

Maternal and child characteristics are summarized in Table 1. Those without DNAm data were more likely to be low SES $(p=0.04)$ and to be a minority race or ethnicity $(p=0.004)$, compared to those with DNAm data. Included and excluded children did not differ based on prenatal risk class or cumulative prenatal risk (all $p>0.05)$.

\section{Prenatal risk}

We first estimated LCA models and used standard model fit statistics to determine the ideal number of latent profiles. Lo-Mendell-Rubin and bootstrapped loglikelihood ratio tests indicated that the 4-profile model did not fit 
Table 1 Demographic and medical characteristics of the sample

\begin{tabular}{|c|c|c|c|c|}
\hline Sample characteristics & $\begin{array}{l}\text { Full sample } \\
(N=601)\end{array}$ & $\begin{array}{l}\text { Included } \\
(N=470)\end{array}$ & $\begin{array}{l}\text { Excluded } \\
(N=131)\end{array}$ & $p$-value \\
\hline \multicolumn{5}{|l|}{ Maternal characteristics* } \\
\hline Maternal education: $<$ HS/GED & $13 \%(79 / 598)$ & $15 \%(68 / 468)$ & $8.5 \%(11 / 130)$ & 0.07 \\
\hline Low SES: Hollingshead $=5$ & $9.9 \%(59 / 599)$ & $8.5 \%(40 / 469)$ & $15 \%(19 / 130)$ & 0.04 \\
\hline Minority race or ethnicity $^{ \pm}$ & $58 \%(347 / 601)$ & $55 \%(257 / 470)$ & $69 \%(90 / 131)$ & 0.004 \\
\hline No partner & $25 \%(152 / 600)$ & $26 \%(124 / 470)$ & $22 \%(28 / 130)$ & 0.26 \\
\hline Neonatal characteristics & $\begin{array}{l}\text { Full sample } \\
(N=704)\end{array}$ & $\begin{array}{l}\text { Included } \\
(N=542)\end{array}$ & $\begin{array}{l}\text { Excluded } \\
(N=162)\end{array}$ & $p$-value \\
\hline Infant gender $=$ Male & $56 \%(388 / 697)$ & $55 \%(299 / 539)$ & $56 \%(89 / 158)$ & 0.85 \\
\hline Multiple gestation & $26 \%(184 / 697)$ & $27 \%(145 / 539)$ & $25 \%(39 / 158)$ & 0.58 \\
\hline Cesarean delivery & $71 \%(495 / 696)$ & $71 \%(382 / 539)$ & $72 \%(113 / 157)$ & 0.79 \\
\hline PMA at Birth (weeks) & $27.0 \pm 1.92$ & $27.0 \pm 1.92$ & $27.0 \pm 1.92$ & 0.86 \\
\hline Birth weight (grams) & $948.3 \pm 280.6$ & $951.1 \pm 281.8$ & $938.5 \pm 277.0$ & 0.62 \\
\hline Head circumference (cm) & $24.5 \pm 2.43$ & $24.5 \pm 2.48$ & $24.4 \pm 2.24$ & 0.70 \\
\hline PMA at Discharge (weeks) & $40.5 \pm 5.43$ & $40.3 \pm 5.20$ & $41.29 \pm 6.12$ & 0.05 \\
\hline Length of NICU stay (LOS days) & $93.5 \pm 41.9$ & $91.7 \pm 40.1$ & $99.7 \pm 47.4$ & 0.05 \\
\hline Weight at discharge (grams) & $3013 \pm 905$ & $3001 \pm 861$ & $3057 \pm 1042$ & 0.50 \\
\hline Severe retinopathy of prematurity & $5.9 \%(41 / 697)$ & $6.3 \%(34 / 539)$ & $4.4 \%(7 / 158)$ & 0.38 \\
\hline Necrotizing enterocolitis/sepsis & $18 \%(128 / 697)$ & $19 \%(103 / 539)$ & $16 \%(25 / 158)$ & 0.35 \\
\hline Chronic lung disease & $51 \%(357 / 697)$ & $51 \%(277 / 539)$ & $51 \%(80 / 158)$ & 0.87 \\
\hline Serious brain injury ${ }^{+}$ & $13 \%(92 / 694)$ & $13 \%(69 / 539)$ & $15 \%(23 / 155)$ & 0.51 \\
\hline
\end{tabular}

Means \pm standard deviations (continuous) or percentage and frequencies (categorical) of demographic and medical characteristics. $p$-values refer to the comparison of included versus excluded individuals and were obtained from t-tests (continuous variables) and chi-squared tests (categorical variables)

PMA, postmenstrual age; HS, high school; GED, General Equivalency Diploma; SES, socioeconomic status

*All mothers with prenatal data were included in the latent class analysis. Included versus excluded in this Table refers to individuals with data for the epigenomewide analysis

${ }^{ \pm}$Minority race or ethnicity was defined as any non-White race (e.g., Black, Asian) or ethnicity (e.g., Hispanic and/or Latino/a)

+ Serious brain injury included parenchymal echodensity, periventricular leukomalacia, or ventricular dilation diagnosed via cranial ultrasound

significantly better than the 3-profile model, but that the 3-profile model did fit significantly better than the 2-profile model. The 3-profile solution had the lowest Bayesian information criterion (BIC), high entropy (0.84), and high class probabilities $(0.89-0.94)$. The class sizes were reasonable (smallest class $=13 \%$ ) and the classes were readily interpretable. Thus, model fit statistics supported a 3-profile LCA solution. Fit statistics for all LCA solutions are included in Additional file 2.

Women in the three latent classes differed on 21 out of 24 prenatal risk factors (Table 2). Figure 1 depicts differences in the 3 classes by rates of endorsement of prenatal risk factors. Women in class 1 ("Typical"; 61\%) had the lowest rates of endorsement for all risk factors. In contrast, women in class 2 ("Physical Risk"; 26\%) exhibited elevated physical health problems, including high rates of obesity, hypertension, and pre-eclampsia. Women in class 3 ("Psychological Risk"; 13\%) exhibited elevated substance use and psychological health problems. They endorsed high rates of alcohol, tobacco, and drug use during pregnancy, as well as high rates of anxiety and depression. Women in class 3 were also more likely to indicate that they felt "down", "slow", and "hopeless" during their pregnancy, and to indicate that their pregnancy was a "very hard time" in their lives.

We then calculated a cumulative prenatal risk score for each participant. On average, mothers experienced an average of 3.6 risk factors $(S D=2.3)$, with a range from 0 to 12 .

\section{Epigenome-wide association study with prenatal risk profiles}

Our first set of models compared DNAm for children born to women in the Physical Risk or Psychological Risk groups to children born to women in the Typical group. Results are displayed in Table 3. After Bonferroni adjustment, one $\mathrm{CpG}$ was differentially methylated in the Physical Risk group and one CpG was differentially methylated in the Psychological Risk group. Compared to the Typical group, neonates of mothers in the Physical Risk group had, on average, $5 \%$ lower DNAm at the identified CpG (cg25123362) located in the body of the BNIP3 
Table 2 Distribution of individual prenatal risk factors in full sample and by latent class

\begin{tabular}{|c|c|c|c|c|c|}
\hline Prenatal risk factors & $\begin{array}{l}\text { Full sample } \\
(N=601)\end{array}$ & $\begin{array}{l}\text { Typical } \\
(N=367 ; 61 \%)\end{array}$ & $\begin{array}{l}\text { Physical risk } \\
(N=155 ; 26 \%)\end{array}$ & $\begin{array}{l}\text { Psychological risk } \\
(N=79 ; 13 \%)\end{array}$ & $x^{2}$ \\
\hline Age $>35$ & $18 \%$ & $18 \%$ & $18 \%$ & $14 \%$ & 0.89 \\
\hline Low SES & $10 \%$ & $6.5 \%$ & $14 \%$ & $18 \%$ & $12.78^{* *}$ \\
\hline$<\mathrm{HS}$ degree & $13 \%$ & $12 \%$ & $9.8 \%$ & $26 \%$ & $12.54^{* *}$ \\
\hline Minority & $58 \%$ & $57 \%$ & $56 \%$ & $66 \%$ & 2.45 \\
\hline No partner & $25 \%$ & $21 \%$ & $18 \%$ & $62 \%$ & $65.33^{* * *}$ \\
\hline No prenatal care & $2.7 \%$ & $1.6 \%$ & $1.9 \%$ & $9.1 \%$ & $14.01 * * *$ \\
\hline Underweight & $5.0 \%$ & $5.8 \%$ & $0.0 \%$ & $12 \%$ & $15.48^{* * *}$ \\
\hline Obese & $34 \%$ & $26 \%$ & $53 \%$ & $33 \%$ & $35.11^{* * *}$ \\
\hline Too much weight gained & $18 \%$ & $12 \%$ & $34 \%$ & $17 \%$ & $33.93^{* * *}$ \\
\hline Hypertension & $27 \%$ & $3.3 \%$ & $95 \%$ & $3.9 \%$ & $490.33^{* * *}$ \\
\hline Pre-eclampsia & $21 \%$ & $0.0 \%$ & $79 \%$ & $0.0 \%$ & $442.57^{* * *}$ \\
\hline Diabetes & $6.0 \%$ & $5.5 \%$ & $9.7 \%$ & $1.3 \%$ & $7.00^{*}$ \\
\hline $\mathrm{STI} / \mathrm{HIV}$ & $7.0 \%$ & $5.0 \%$ & $6.5 \%$ & $18 \%$ & $16.33^{* * *}$ \\
\hline Infection & $10 \%$ & $10 \%$ & $9.0 \%$ & $14 \%$ & 1.39 \\
\hline Alcohol & $3.2 \%$ & $1.4 \%$ & $2.6 \%$ & $13 \%$ & $27.18^{* * *}$ \\
\hline Illegal substances & $4.5 \%$ & $0.3 \%$ & $4.5 \%$ & $24 \%$ & $85.30^{* * *}$ \\
\hline Tobacco & $14 \%$ & $8.0 \%$ & $14 \%$ & $44 \%$ & $70.36^{* * *}$ \\
\hline Marijuana & $10 \%$ & $4.1 \%$ & $8.4 \%$ & $41 \%$ & $96.07^{* * *}$ \\
\hline Depression & $11 \%$ & $4.9 \%$ & $10 \%$ & $39 \%$ & $79.51^{* * *}$ \\
\hline Anxiety & $12 \%$ & $7.4 \%$ & $9.7 \%$ & $35 \%$ & $50.62^{* * *}$ \\
\hline Pregnancy"Hard Time" & $11 \%$ & $6.7 \%$ & $6.5 \%$ & $42 \%$ & $86.18^{* * *}$ \\
\hline Pregnancy "Felt Down" & $9.9 \%$ & $2.7 \%$ & $5.9 \%$ & $51 \%$ & $173.87^{* * *}$ \\
\hline Pregnancy"Felt Slow" & $20 \%$ & $13 \%$ & $23 \%$ & $46 \%$ & $44.90^{* * *}$ \\
\hline Pregnancy"Felt Hopeless" & $3.9 \%$ & $0.3 \%$ & $1.3 \%$ & $26 \%$ & $115.30^{* * *}$ \\
\hline
\end{tabular}

STI, sexually transmitted infection; HIV, Human immunodeficiency virus ${ }^{*} p<.05,{ }^{* *} p<.01,{ }^{* * *} p<.001$

gene. Neonates of mothers in the Psychological Risk had 4\% lower DNAm at the identified CpG (cg08930413) located in the body of the PRKAG2 gene.

\section{Epigenome-wide association study with cumulative prenatal risk}

Next, we tested for associations between cumulative prenatal risk and neonatal DNAm. We found 15 statistically significant CpGs that were differentially methylated with increasing cumulative prenatal risk (Fig. 2). Increasing prenatal risk was associated with lower DNAm at 12 CpGs and higher DNAm at 3 CpGs. These differences were small in magnitude and ranged from a $1-6 \%$ difference in DNAm associated with a $10 \%$ increase in cumulative prenatal risk.

\section{Brain-buccal correlations}

We used the publicly available IMAGE-CpG website (http://han-lab.org/methylation/default/imageCpG) to explore whether DNAm levels in buccal tissue was correlated with DNAm levels in brain tissue [46], for the CpGs that were identified as significant in our EWAS models. While neither of the CpGs identified in our prenatal risk profile EWAS exhibited significant brain-buccal correlations, 4 of the $15 \mathrm{CpGs}$ that were identified in our models of cumulative prenatal risk did exhibit significant brainbuccal correlations $(r=0.44$ to $0.61, p<0.05)$.

\section{Functional and phenotypic enrichment}

Because few CpGs were significant in our prenatal risk profile EWAS, we considered in our enrichment analyses those CpGs that were significant within a 5\% FDR in the cumulative prenatal risk model ( $n=384 \mathrm{CpGs})$. After FDR correction, there were no significantly enriched pathway or gene ontology terms.

\section{CpG annotation}

We identified phenotypes and traits that have been associated with the genes annotated to significant CpGs in our EWAS models (Table 4). Of the 17 significant CpGs, 9 were located in genes associated with neurobehavioral traits including cognitive ability, memory, reaction time, 


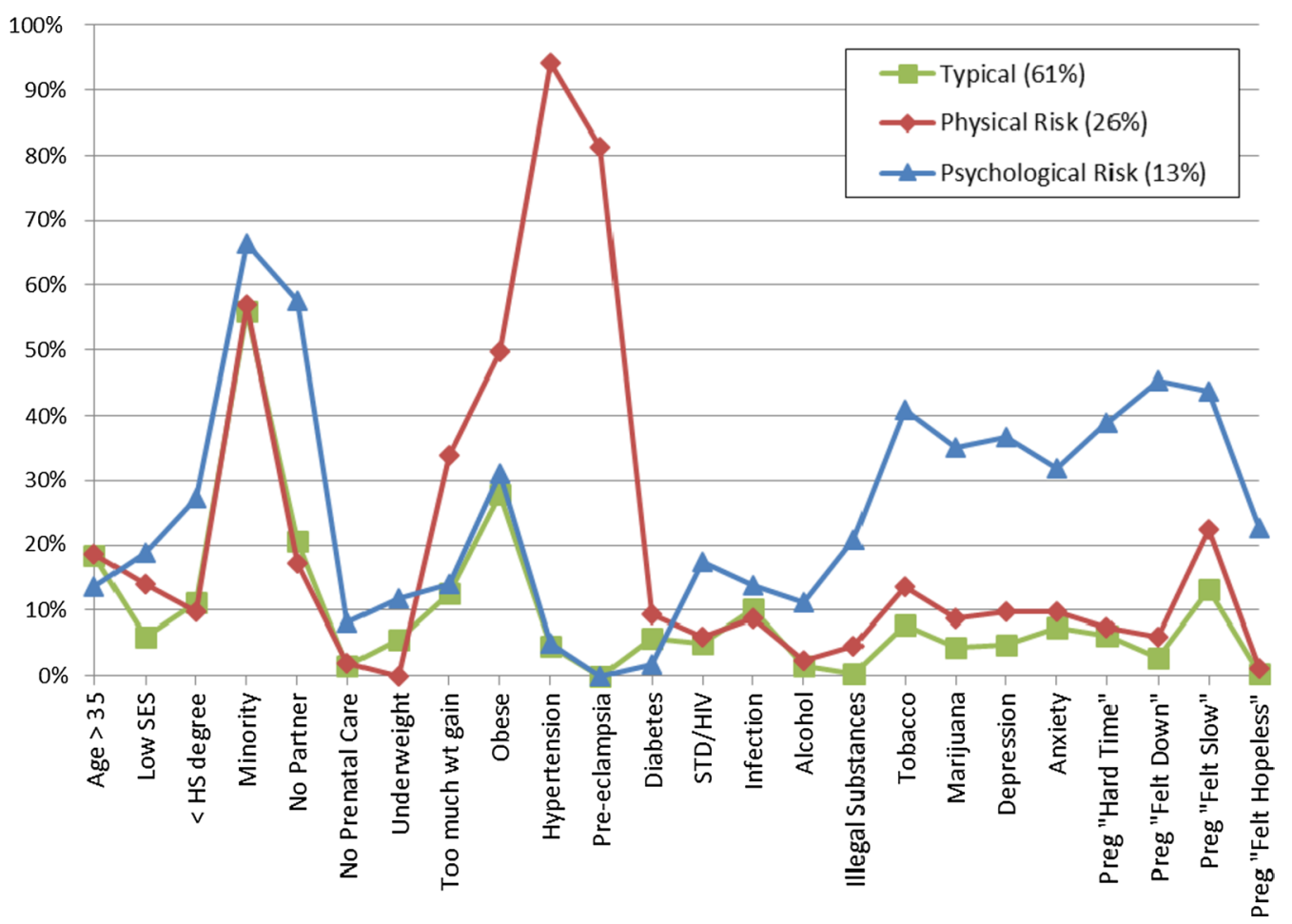

Fig. 1 Rates of endorsement of 24 prenatal risk factors by latent class membership. Women in class 1 (green; $61 \%$ ) endorse few prenatal risk factors. Women in class 2 (red; 26\%) endorse elevated physical health problems, whereas women in class 3 (blue; 13\%) endorse elevated substance use and psychological problems

brain volume, and mental health disorders (e.g., depression, bipolar disorder, schizophrenia). Of these 9 CpGs within neurobehaviorally-linked genes, 3 had significant blood-buccal correlations (cg19573457 [CRYBB2P1], cg11221492 [MBIP], cg22102865 [ZNF398]).

Additionally, 11 of the 17 CpGs were located in genes associated with physical health markers, including body mass index, cardiovascular disease, hypertension, type-2 diabetes, and white blood cell counts. Of these $11 \mathrm{CpGs}$ within health-linked genes, 3 had significant blood-buccal correlations (cg16999677 [ZDHHC11], cg11221492 [MBIP], cg22102865 [ZNF398]).

\section{DMR analysis}

Last, we performed DMR analyses to test whether there were regional, not just $\mathrm{CpG}$ site-specific, differences in DNAm associated with prenatal risk factors. DMR analyses comparing the Physical Risk group to the Typical group found one significant region (Chr10: 133793734133794558) containing three CpG sites (cg25123362, cg12751948, cg16592121). Children born to mothers in the Physical Risk group had less methylation in this region, on average, compared to children born to mothers in the Typical group $(p=4.17 \mathrm{E}-11)$. This region contained the individually significant $\mathrm{CpG}$ described above in the Physical Risk versus Typical model (cg25123362) and this DMR similarly annotated to the BNIP3 gene.

Analyses comparing the Psychological Risk group to the Typical group also resulted in one significant DMR (Chr14: 77785784-77785968) containing two CpG sites (cg02181287, cg03738767). Children born to mothers in the Psychological Risk group had less methylation in this region, on average, compared to children born to mothers in the Typical group ( $p=4.65 \mathrm{E}-08)$. This DMR was in a different genomic location compared to the individually significant $\mathrm{CpG}$ described earlier and annotated to the GSTZ1 and POMT2 genes.

Finally, we found six DMRs that were significantly associated with cumulative prenatal risk. Five of the six were negatively associated with prenatal risk, suggesting lower DNAm with increasing levels of risk. One DMR was positively associated, suggesting more DNAm with increasing levels of risk. One of the six significant DMRs (Chr7: 148843026-148844053) was in a similar region as a CpG (cg22102865) that was identified as being individually significant. One region (Chr14: 7778578477785968) that was identified as a DMR related to cumulative prenatal risk was also identified as a significant DMR in the comparison of the Psychological Risk group to the Typical group. The other four DMRs (Chr3: 
Table 3 Epigenome-wide association study results for CpG sites that yielded significant associations after Bonferroni adjustment

\begin{tabular}{|c|c|c|c|c|c|c|c|}
\hline CpG & Location & Gene annotation & $\begin{array}{l}\text { Coefficient } \\
\text { (m-value) }\end{array}$ & $p$ value (Raw) & $p$ value (Adj) & $\begin{array}{l}\text { Coefficient }^{\mathrm{a}} \\
\text { (beta-value) }\end{array}$ & $\begin{array}{l}\text { Brain-buccal } \\
\text { correlation }\end{array}$ \\
\hline \multicolumn{8}{|c|}{$\begin{array}{l}\text { Model 1: Physical Risk vs. } \\
\text { Typical }\end{array}$} \\
\hline $\operatorname{cg} 25123362$ & Chr10: 133793734 & BNIP3 (Body) & -0.25 & $2.76 \mathrm{E}-11$ & $1.95 \mathrm{E}-05$ & -0.05 & -0.09 \\
\hline \multicolumn{8}{|c|}{$\begin{array}{l}\text { Model 1: Psychological Risk } \\
\text { vs. Typical }\end{array}$} \\
\hline cg08930413 & Chr7: 151548036 & PRKAG2 (Body) & -0.28 & $2.11 \mathrm{E}-08$ & $1.49 \mathrm{E}-02$ & -0.04 & 0.22 \\
\hline \multicolumn{8}{|c|}{$\begin{array}{l}\text { Model 2: Cumulative Prena- } \\
\text { tal Risk }\end{array}$} \\
\hline cg16999677 & Chr5: 843982 & ZDHHC11 (Body) & -2.05 & $6.85 \mathrm{E}-08$ & $4.84 \mathrm{E}-02$ & -0.05 & $0.61^{* *}$ \\
\hline cg05324191 & Chr1: 116994757 & LOC101929023 (Body) & 1.03 & $1.30 \mathrm{E}-09$ & $9.18 \mathrm{E}-04$ & 0.02 & -0.33 \\
\hline cg01533736 & Chr20: 22542854 & LINC00261 (Body) & -1.18 & 1.17E-09 & $8.25 \mathrm{E}-04$ & -0.03 & 0.40 \\
\hline cg00569188 & Chr21: 41122530 & IGSF5 (Body) & -1.28 & $4.61 \mathrm{E}-08$ & $3.26 \mathrm{E}-02$ & -0.03 & -0.17 \\
\hline cg09979763 & Chr1: 245499904 & KIF26B (Body) & -2.02 & $4.23 \mathrm{E}-08$ & $2.99 \mathrm{E}-02$ & -0.03 & -0.07 \\
\hline cg11420269 & Chr16: 70516713 & COG4 (Body; ExonBnd) & -1.46 & 2.07E-08 & $1.46 \mathrm{E}-02$ & -0.03 & 0.02 \\
\hline cg27514986 & Chr15: 39486981 & C15orf5 $4^{+}$ & 2.78 & $9.74 \mathrm{E}-09$ & $6.88 \mathrm{E}-03$ & 0.05 & 0.19 \\
\hline cg05636131 & Chr7: 148844053 & ZNF398 (5'UTR; TSS1500) & -1.09 & $6.93 \mathrm{E}-08$ & $4.90 E-02$ & -0.02 & $0.42^{\ddagger}$ \\
\hline $\operatorname{cg} 11531492$ & Chr3: 125673505 & ROPN1B $B^{+}$ & -2.63 & $6.56 \mathrm{E}-08$ & $4.63 \mathrm{E}-02$ & -0.05 & -0.08 \\
\hline cg26760502 & Chr14: 105493800 & $\mathrm{CDCA4}^{+}$ & -0.88 & $4.89 \mathrm{E}-08$ & $3.45 E-02$ & -0.01 & -0.09 \\
\hline cg19573457 & Chr22: 25893657 & $C R Y B B 2 P 1^{+}$ & -1.68 & $2.17 \mathrm{E}-08$ & $1.53 \mathrm{E}-02$ & -0.04 & $0.44^{*}$ \\
\hline cg01284858 & Chr10: 123902371 & TACC2 (5'UTR; Body) & 3.61 & $6.20 E-09$ & $4.38 \mathrm{E}-03$ & 0.06 & -0.32 \\
\hline $\operatorname{cg} 12155575$ & Chr3: 186965150 & MASP1 (Body) & -1.16 & 8.97E-09 & $6.33 \mathrm{E}-03$ & -0.03 & 0.08 \\
\hline $\operatorname{cg} 11221492$ & Chr14: 36790270 & MBIP (TSS1500) & -0.90 & $3.92 \mathrm{E}-08$ & $2.77 \mathrm{E}-02$ & -0.01 & $0.54^{*}$ \\
\hline $\operatorname{cg} 22102865$ & Chr7: 148844067 & ZNF398 (TSS1500; 5'UTR) & -1.24 & $1.37 \mathrm{E}-08$ & $9.64 \mathrm{E}-03$ & -0.02 & $0.46^{*}$ \\
\hline
\end{tabular}

${ }^{a}$ Note that the coefficient for cumulative risk models represents the expected increase in \% DNAm associated with a $10 \%$ increase in risk. ${ }^{*} p<.10,{ }^{*} p<.05,{ }^{* *} p<.01,{ }^{+}$ indicates closest gene. Adjusted $p$-value is Bonferroni corrected

186965021-186965150; Chr1: 155659719-155659882; Chr22: 25884154-25884537; Chr15: 3426071234260956) did not share CpGs in common with other DMR analyses or with the individual CpG results. These four DMRs annotated to the following genes: MASP1, $D A P 3, Y Y 1 A P 1, C R Y B B 2 P 1, A V E N$, and CHRM5. Full results for the DMR analysis are included in Additional file 3.

\section{Discussion}

We conducted an epigenome-wide study to test the associations between prenatal risk factors and neonatal DNAm in a sample of very preterm neonates. LCA findings showed 3 distinct prenatal risk groups; a group with few risk factors ("Typical"; 61\%) and groups with elevated physical $(26 \%)$ or psychological $(13 \%)$ risk factors. Neonates born to women in these higher risk subgroups had differential DNAm patterns at two CpG sites. The cumulative prenatal risk analysis showed that a higher risk score was associated with greater methylation at $3 \mathrm{CpG}$ sites and lower DNAm at $12 \mathrm{CpG}$ sites.

The investigation of both the total number (cumulative score) and co-occurring types (LCA profiles) of prenatal risk factors as they relate to epigenomewide DNAm in infants, including preterm infants, is novel. Previous EWAS have relied on a single variable approach with mixed findings. Two studies found no associations between psychological risk factors and neonatal DNAm [24, 25], several studies uncovered only a small number of differentially methylated CpGs [26-31], and one study found 145 differentially methylated CpGs [51]. The only other study using a cumulative risk approach found no significant associations between cumulative prenatal stress and neonatal DNAm [24]. However, this study measured DNAm from blood, used a different DNAm bead chip, included term as well as preterm children, and did not assess physical health risks. In contrast, the cumulative prenatal risk index used in the current study accounted for 24 demographic, substance use, physical health, and psychological health indicators. There was no overlap between significant CpGs or genes identified in this study and those identified in any of the previous EWAS on prenatal risk factors. These differences may be due to differences in methods (e.g., which risk factors were assessed; single versus multiple risk approach) and 


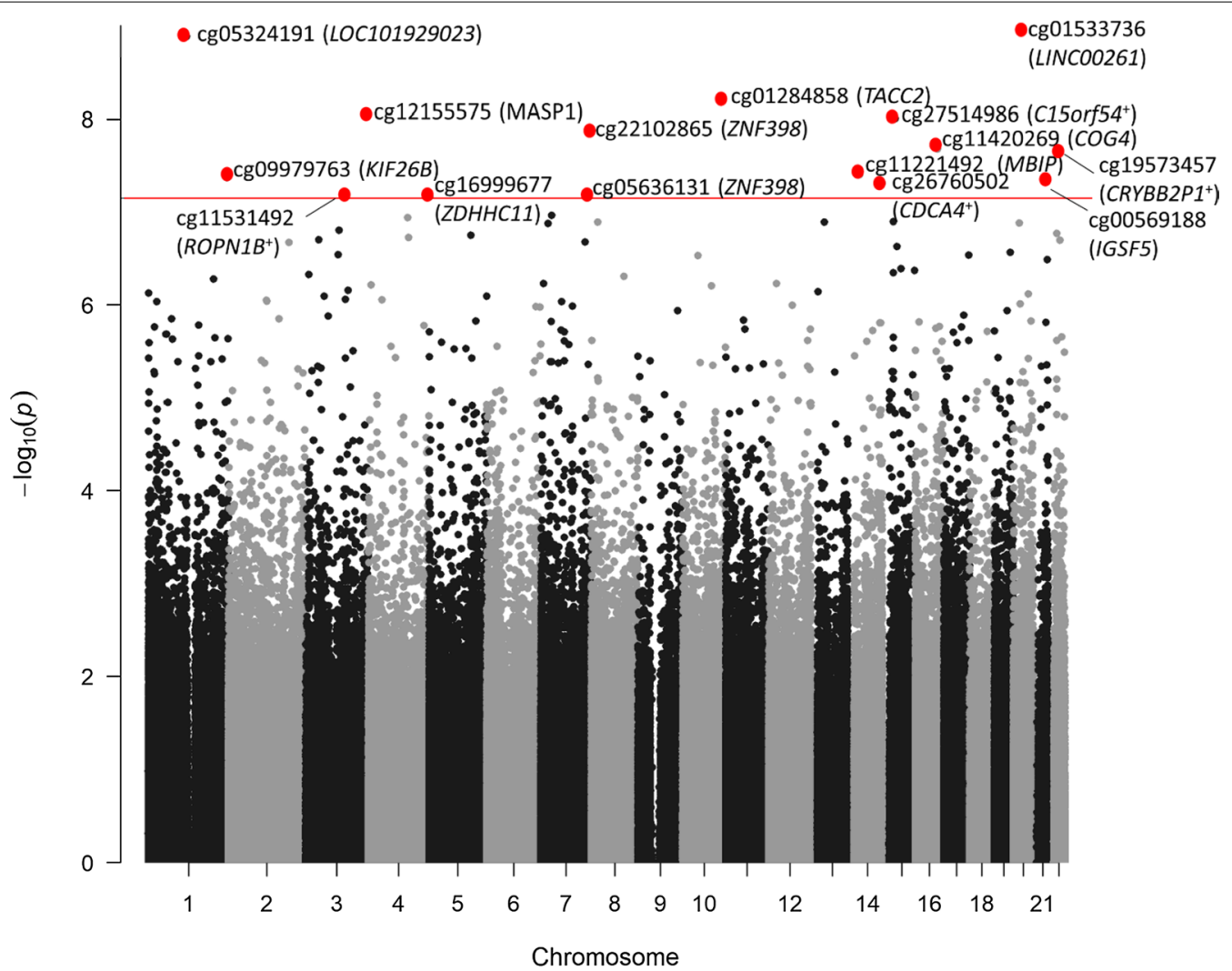

Fig. 2 Manhattan plot of epigenetic loci associated with cumulative prenatal risk. The $x$-axis shows the genomic location of individual $C p G$ sites and the $y$-axis shows the $-\log _{10}(p$ values) from models relating cumulative prenatal risk to $\mathrm{CpG}$ methylation, adjusting for child sex, recruitment site, postmenstrual age at collection, sample batch, and cellular heterogeneity. Gene annotations have been added for all CpGs yielding significant associations after Bonferroni adjustment. The horizontal red line depicts the Bonferroni adjusted $p$-value threshold $(a=0.05 / 706323)$. ${ }^{+}$indicates closest gene

samples (e.g., primarily term versus exclusively preterm children).

We also investigated the relationship between prenatal stress phenotypes and neonatal DNAm by using LCA to classify women into subgroups on the basis of the same 24 risk factors. We found evidence for three distinct prenatal stress phenotypes. The majority of women belonged to a group that experienced few risk factors. In comparison, women in the physical risk group had elevated levels of medical risk factors, such as hypertension (95\%) and pre-eclampsia (79\%). Women in the psychological risk group had elevated levels of mental health concerns, including the highest rates of depression (39\%) and anxiety (35\%), as well as the highest rates of tobacco (44\%), marijuana (41\%), alcohol (13\%), and illegal drug (24\%) use. Previous work investigating prenatal stress phenotypes in relation to fetal and neonatal behavior [52] also found a group with elevated physical risk factors (e.g., higher blood pressure, greater calorie, fat, and sugar consumption) and a group with elevated psychological risk factors (e.g., greater depression, anxiety, and perceived stress) with similar proportions of women falling into the low risk or typical group (approximately $2 / 3$ ) versus one of the two higher risk groups (approximately $1 / 3$ ). These similarities emerged despite different study methodologies (e.g., maternal self-reported versus objectively assessed risk factors) and different variables included in the latent models. Taken together, these findings provide evidence for distinct subgroups of women who may differentially be impacted by physical health issues or psychological health issues during pregnancy. However, our study is the first to demonstrate associations between prenatal risk phenotypes and neonatal DNAm.

Among the $15 \mathrm{CpGs}$ that were associated with cumulative prenatal risk, 12 were located in genes that have been linked in GWAS studies to relevant phenotypes for both physical (e.g., blood pressure [53], BMI [54], diabetes [55]) and mental health outcomes (e.g., schizophrenia [56], depression [57], bipolar disorder [58]) as well as neurodevelopmental markers (e.g., brain volume/measurement [59], reaction time [60]). One gene $(C D C A 4)$ identified in our analysis encodes a member 
Table 4 CpGs associated with prenatal risk are linked to genes that have been associated with traits in the GWASdb

\begin{tabular}{|c|c|c|}
\hline Gene & Traits (N) & Selected traits \\
\hline BNIP3 & 8 & $\begin{array}{l}\text { Self-reported educational attainment, intelligence, mathematical ability, household income, schizophrenia, cognitive func- } \\
\text { tion }\end{array}$ \\
\hline PRKAG2 & 38 & $\begin{array}{l}\text { Self-reported educational attainment, mathematical ability, brain/neuroimaging measurement, gut microbiome meas- } \\
\text { urement, white matter microstructure, cardiovascular disease, brain volume measurement, bipolar disorder, psychotic } \\
\text { symptoms, cognitive function }\end{array}$ \\
\hline ZDHHC11 & 1 & Myopia age of onset \\
\hline LOC101929023 & - & - \\
\hline LINC00261 & 4 & Body mass index, fasting blood glucose measurement, birth weight \\
\hline IGSF5 & 4 & Systolic blood pressure, hypertension, short-term memory, health literacy \\
\hline KIF26B & 15 & $\begin{array}{l}\text { Brain volume/neuroimaging measurement, response to SSRI, unipolar depression, diet measurement, brain measurement, } \\
\text { schizophrenia }\end{array}$ \\
\hline COG4 & 3 & Body height, body weight, body mass index \\
\hline C15orf54 & 1 & Dihydroxy docosatrienoic acid measurement \\
\hline ZNF398 & 10 & Brain volume/neuroimaging measurement, white blood cell count \\
\hline ROPNIB & - & - \\
\hline CDCA4 & 1 & Telomere length \\
\hline CRYBB2P1 & 4 & Bipolar disorder \\
\hline TACC2 & 16 & Opioid dependence, metabolite measurement, body weight gain, schizophrenia, body height, reaction time measurement \\
\hline MASP1 & 3 & Type 2 diabetes \\
\hline MBIP & 6 & Self-reported educational attainment, hypertension \\
\hline
\end{tabular}

of the E2F family of transcription factors, which regulate spindle organization, cytokinesis, and cell proliferation [61]. This gene has also previously been shown to be associated with leukocyte telomere length [62], suggesting potential ties between prenatal risk and biological aging processes. There was also some overlap in genes (KIF26B; TACC2) identified in the current analysis with genes we previously reported to be associated $($ FDR $<0.10)$ with atypical neurodevelopmental profiles in the same sample [45]. Therefore, it is possible that DNAm of these genes may play a role in explaining the prenatal programming of child neurodevelopment, although additional longitudinal studies would be needed to rigorously test this hypothesis.

Only 2 CpG sites were differentially methylated across prenatal risk groups. Neonates of mothers in the physical risk group had $5 \%$ less methylation, on average, at cg25123362, located in the BNIP3 gene. Neonates of mothers in the psychological risk group had 4\% less methylation, on average, at cg08930413, located in the PRKAG2 gene. Interestingly, both the BNIP3 and PRKAG2 genes have been associated with similar traits in previous GWAS analyses, including educational attainment [63] and cognitive function [60, 63], suggesting that the associations between prenatal risk factors and child outcomes may be marked by some degree of equifinality (i.e., different biological pathways leading to the same outcome) [64].
Our DMR analyses yielded overlapping results with the individual CpG analyses for ZNF398 (associated with cumulative risk) and BNIP3 (associated with physical risk group). We also identified four significant DMRs that did not include individually significant CpGs from our EWAS, annotated to MASP1, DAP3, YY1AP1, CRYBB2P1, AVEN, and CHRM5. ZNF398 and BNIP3 are particularly interesting given that they were identified in both CpG-specific and regional analyses. Prior GWAS have linked ZNF398 to brain volume and neuroimaging measurements [65], while BNIP3 has been linked to cognitive function [60]. Additionally, we found that DNAm levels at one CpG within the ZNF398 gene (cg22102865) were positively correlated between brain and buccal tissues from publicly available data [46].

It was notable that our analysis using cumulative prenatal risk identified more significant CpGs $(N=15)$ than our analyses investigating phenotypes of prenatal risk $(N=2)$. There was also no overlap in significant CpGs or genes identified by the two models, suggesting that they may be unique methods for identifying risk. Cumulative risk models are attractive because of their simplicity, parsimony, and relatively greater statistical power, compared to alternative approaches (e.g., individual risk variables) [66]. They also mimic how these factors impact pregnant women as they rarely occur in isolation. An empirical comparison of cumulative risk indices to either individual variable or factor score approaches 
also found that risk indices provide better prediction of developmental patterns [67]. Therefore, cumulative prenatal risk indices may be a useful approach in that they provide a strong signal for the relationship of early adversity to child outcomes, including DNAm. Indeed, there is precedence in the epigenetic literature for the use of cumulative risk scores as predictors of children's DNAm $[24,68]$. The disadvantages of cumulative risk indices are that each individual risk factor carries equal weight. Moreover, we cannot determine which variables are the most important drivers in explaining the association with outcomes which limits the practical application of these risk indices in clinical practice. Alternative "person-centered" approaches, like LCA and LPA models, allow for the modeling of patterns of correlated risk factors as they co-occur in real participants. An underlying assumption is that different types of risk are differentially associated with outcomes. "Person-centered" approaches offer advantages over individual variable or cumulative risk approaches in that they are both comprehensive and specific. The differentiation of prenatal risk phenotypes into physical and psychologically stressed individuals offer a new way to think about types of adverse prenatal environments that may be differentially related to child outcomes [52] and may require different types of intervention. Previous studies comparing cumulative risk and LPA approaches in the context of child development have similarly reported that these two types of analyses provide complementary information about the relationships between risk factors and outcomes [69].

Limitations of this study are to be appreciated. First, we only considered binary risk factors as opposed to continuous indicators. This decision was partly necessitated by our creation of a cumulative risk index. Many of the risk factors we included (e.g., presence or absence of physical or mental health diagnosis) are dichotomous in nature but some loss of information may have occurred from dichotomizing other variables (e.g., SES). Second, as the inclusion criteria for this study included birth prior to 30 weeks gestation and likely survival to discharge, women were necessarily recruited after pregnancy, and some pregnancy data were assessed retrospectively (e.g., maternal report of pregnancy moods and feelings). However, any retrospective data were collected in the neonatal period, potentially reducing the impact of recall bias. Third, we were unable to locate an external replication sample because of the unique nature of this cohort (e.g., very preterm neonates). The unique nature of the sample means that it is unclear to what extent our results are sample specific or whether they would generalize to later preterm or term children. Fourth, we observed differential DNAm in buccal cells rather than in the tissues that may be more clearly related to children's health (i.e., neural tissues for neurodevelopment). However, a benefit of measuring DNAm in peripheral tissue is that it could represent processes that are occurring elsewhere in the body such as in the immune and metabolic systems. As prematurity is a systemic condition impacting nearly all organ systems, peripheral tissues may be particularly relevant to study in this sample. Finally, although the differences in DNAm we observed were small (1-6\%), they are consistent with what has been reported in other epidemiological studies investigating peripheral DNAm as it relates to other prenatal risk factors (e.g., smoking [70]), as well as previous studies in our sample [37, 45]. However, small effects in DNAm are likely important [71], as they open a potential window into understanding mechanisms driving child health.

\section{Conclusions}

In sum, we observed associations between prenatal risk factors and DNAm in very preterm infants using both cumulative risk and risk phenotype approaches. Epigenetics offers a potential biological indicator of the amount and type of prenatal risk that children were exposed to, which may be particularly useful for identifying infants at greatest risk especially in populations of vulnerable infants. There remains a need to better understand whether differences in DNAm at birth are related to children's health and neurodevelopmental trajectories.

\section{Abbreviations}

CpG: Cytosine-phosphate-guanine; DNAm: DNA methylation; DMR: Differentially methylated regions; EWAS: Epigenome-wide association study; GEE: Generalized estimating equations; GO: Gene ontology; LCA: Latent class analysis; LPA: Latent profile analysis; NOVI: Neonatal Neurobehavior and Outcomes in Very Preterm Infants; PMA: Postmenstrual age.

\section{Supplementary Information}

The online version contains supplementary material available at https://doi. org/10.1186/s13148-021-01164-9.

Additional file 1. EWAS results for all suggestive associations (FDR < 10\%). This Excel spreadsheet contains information about all CpGs with FDR $<10 \%$ from the models comparing physical risk to typical (Model 1), psychological risk to typical (Model 2), and cumulative risk (Model 3).

Additional file 2. Fit statistics for latent class analysis of prenatal risk factors. This table presents model fit statistics used to choose the optimal number of profiles in the latent class analysis.

Additional file 3. Differentially methylated regions (DMR) associated with prenatal risk. This table presents results from the results of the DMR analysis, including the location, estimates, $p$-values, and gene annotations associated with all significant DMRs.

\section{Acknowledgements}

Not applicable.

\section{Authors' contributions}

MC, SG, TME, JAH, TMO, CJM, and BML conceptualized and designed the study. SAD coordinated data collection. LMD prepared data. MC, SG, TME, and BML 
analyzed data and drafted the article. MC, SG, TME, ECM, JAH, TMO, BSC, JBH, $J C, C R N, S L P, L M S, L M D, S A D, C J M$, and BML contributed to interpretation of results and revisions to the manuscripts. All authors read and approved the final manuscript.

\section{Funding}

This work was funded by the National Institutes of Health (NIH)/Eunice Kennedy Shriver National Institute of Child Health and Human Development (NICHD) grant R01HD072267 (Lester and O'Shea) and R01HD084515 (Lester and Everson). Dr. Camerota was additionally supported by an institutional training grant from the National Institutes of Mental Health (NIMH), grant T32MH019927.

\section{Availability of data and materials}

The raw and processed DNAm data are publicly accessible through NCBI Gene Expression Omnibus (GEO) via accession series GSE128821.

\section{Declarations}

\section{Ethics approval and consent to participate}

All participating mothers provided written informed consent and enrollment and consent procedures were approved by the institutional review boards of Women and Infants Hospital, Spectrum Health, Children's Mercy Office of Research Integrity, Wake Forest University Health Sciences, John F. Wolf, MD Human Subjects Committee at the Lundquist Institute Los Angeles BioMed, Emory University and Western Institutional Review Board (WIRB).

\section{Consent for publication}

Not applicable.

\section{Competing interests}

The authors declare that they have no competing interests.

\section{Author details}

${ }^{1}$ Department of Psychiatry and Human Behavior, Alpert Medical School of Brown University, Providence, RI, USA. ${ }^{2}$ Department of Pediatrics, Women and Infants Hospital of Rhode Island, 101 Dudley Street, Providence, RI 02905, USA. ${ }^{3}$ Gangarosa Department of Environmental Health, Emory University Rollins School of Public Health, Atlanta, GA, USA. ${ }^{4}$ Department of Epidemiology, Emory University Rollins School of Public Health, Atlanta, GA, USA. ${ }^{5}$ Department of Pediatrics, Alpert Medical School of Brown University, Providence, RI, USA. ${ }^{6}$ Department of Pediatrics, University of North Carolina at Chapel Hill School of Medicine, Chapel Hill, NC, USA. ${ }^{7}$ Department of Pediatrics-Neonatology, Children's Mercy Hospital, Kansas City, MO, USA. ${ }^{8}$ Department of Pediatrics, Wake Forest School of Medicine, Winston-Salem, NC, USA. ${ }^{9}$ Department of Pediatrics, University of Hawaii John A. Burns School of Medicine, Honolulu, HI, USA. ${ }^{10}$ Department of Pediatrics, Spectrum Health-Helen DeVos Hospital, Grand Rapids, MI, USA. ${ }^{11}$ Department of Pediatrics, Harbor-UCLA Medical Center, Torrance, CA, USA.

Received: 28 May 2021 Accepted: 2 September 2021 Published online: 10 September 2021

\section{References}

1. Johnson S, Marlow N. Preterm birth and childhood psychiatric disorders. Pediatr Res. 2011;69:22-8.

2. Stephens BE, Vohr BR. Neurodevelopmental outcome of the premature infant. Pediatr Clin North Am. 2009;56:631-46.

3. Chung EH, Chou J, Brown KA. Neurodevelopmental outcomes of preterm infants: a recent literature review. Transl Pediatr. 2020;9:S3-8.

4. Johnson S, Marlow N. Early and long-term outcome of infants born extremely preterm. Arch Dis Child. 2017;102:97-102.

5. Aarnoudse-Moens CSH, Weisglas-Kuperus N, van Goudoever JB, Oosterlaan J. Meta-analysis of neurobehavioral outcomes in very preterm and/ or very low birth weight children. Pediatrics. 2009;124:717-28.

6. Aylward GP. Neurodevelopmental outcomes of infants born prematurely. J Dev Behav Pediatr. 2014;35:394-407.
7. Rysavy MA, Colaizy TT, Bann CM, DeMauro SB, Duncan AF, Brumbaugh JE, et al. The relationship of neurodevelopmental impairment to concurrent early childhood outcomes of extremely preterm infants. J Perinatol. 2021;23:1-9. https://doi.org/10.1038/s41372-021-00999-7.

8. Cserjesi R, Van Braeckel KNJA, Timmerman M, Butcher PR, Kerstjens JM, Reijneveld SA, et al. Patterns of functioning and predictive factors in children born moderately preterm or at term. Dev Med Child Neurol. 2012;54:710-5.

9. Heeren T, Joseph RM, Allred EN, O'Shea TM, Leviton A, Kuban KCK. Cognitive functioning at the age of 10 years among children born extremely preterm: a latent profile approach. Pediatr Res. 2017;82:614-9. https://doi. org/10.1038/pr.2017.82

10. Burnett AC, Youssef G, Anderson PJ, Duff J, Doyle LW, Cheong JLY. Exploring the "preterm behavioral phenotype" in children born extremely preterm. J Dev Behav Pediatr. 2019;40:200-7.

11. Hofheimer JA, Smith LM, McGowan EC, O'Shea TM, Carter BS, Neal CR, et al. Psychosocial and medical adversity associated with neonatal neurobehavior in infants born before 30 weeks gestation. Pediatr Res. 2020;87:721-9. https://doi.org/10.1038/s41390-019-0607-1.

12. Liu J, Bann C, Lester B, Tronick E, Das A, Lagasse L, et al. Neonatal neurobehavior predicts medical and behavioral outcome. Pediatrics. 2010;125:e90-8.

13. McGowan EC, Hofheimer JA, O'Shea TM, Carter BS, Helderman J, Neal CR, et al. Sociodemographic and medical influences on neurobehavioral patterns in preterm infants: a multi-center study. Early Hum Dev. 2020;142: 104954. https://doi.org/10.1016/j.earlhumdev.2020.104954.

14. Conradt E, Adkins DE, Crowell SE, Raby KL, Diamond LM, Ellis B. Incorporating epigenetic mechanisms to advance fetal programming theories. Dev Psychopathol. 2018;30:807-24.

15. Oberlander TF, Weinberg J, Papsdorf M, Grunau R, Misri S, Devlin AM. Prenatal exposure to maternal depression, neonatal methylation of human glucocorticoid receptor gene (NR3C1) and infant cortisol stress responses. Epigenetics. 2008;3:97-106.

16. Conradt E, Hawes K, Guerin D, Armstrong DA, Marsit CJ, Tronick E, et al. The contributions of maternal sensitivity and maternal depressive symptoms to epigenetic processes and neuroendocrine functioning. Child Dev. 2016;87:73-85.

17. Lester BM, Conradt E, LaGasse LL, Tronick EZ, Padbury JF, Marsit CJ. Epigenetic programming by maternal behavior in the human infant. Pediatrics. 2018;142: e20171890. https://doi.org/10.1542/peds.2017-1890.

18. Lester BM, Conradt E, Marsit C. Introduction to the special section on epigenetics. Child Dev. 2016;87:29-37. https://doi.org/10.1111/cdev.12489.

19. Monk C, Spicer J, Champagne FA. Linking prenatal maternal adversity to developmental outcomes in infants: the role of epigenetic pathways. Dev Psychopathol. 2012;24:1361-76.

20. Gartstein MA, Skinner MK. Prenatal influences on temperament development: the role of environmental epigenetics. Dev Psychopathol. 2018;30:1269-303.

21. Robinson R, Lahti-Pulkkinen M, Heinonen K, Reynolds RM, Räikkönen K. Fetal programming of neuropsychiatric disorders by maternal pregnancy depression: a systematic mini review. Pediatr Res. 2019;85:134-45. https:// doi.org/10.1038/s41390-018-0173-y.

22. Ryan J, Mansell T, Fransquet P, Saffery R. Does maternal mental wellbeing in pregnancy impact the early human epigenome? Epigenomics. 2017;9:313-32. https://doi.org/10.2217/epi-2016-0118.

23. Nowak AL, Anderson CM, Mackos AR, Neiman E, Gillespie SL. Stress during pregnancy and epigenetic modifications to offspring DNA. J Perinat Neonatal Nurs. 2020;34:134-45. https://doi.org/10.1097/JPN.0000000000 000471.

24. Rijlaarsdam J, Pappa I, Walton E, Bakermans-Kranenburg MJ, Mileva-Seitz VR, Rippe RCA, et al. An epigenome-wide association meta-analysis of prenatal maternal stress in neonates: a model approach for replication. Epigenetics. 2016;11:140-9. https://doi.org/10.1080/15592294.2016. 1145329.

25. Rodney NC, Mulligan CJ. A biocultural study of the effects of maternal stress on mother and newborn health in the Democratic Republic of Congo. Am J Phys Anthropol. 2014;155:200-9. https://doi.org/10.1002/ ajpa.22568.

26. Cardenas A, Faleschini S, Cortes Hidalgo A, Rifas-Shiman SL, Baccarelli AA, DeMeo DL, et al. Prenatal maternal antidepressants, anxiety, and depression and offspring DNA methylation: epigenome-wide associations at 
birth and persistence into early childhood. Clin Epigenetics. 2019;11:56. https://doi.org/10.1186/s13148-019-0653-x.

27. Gurnot C, Martin-Subero I, Mah SM, Weikum W, Goodman SJ, Brain U, et al. Prenatal antidepressant exposure associated with CYP2E1 DNA methylation change in neonates. Epigenetics. 2015;10:361-72. https:// doi.org/10.1080/15592294.2015.1026031.

28. Non AL, Binder AM, Kubzansky LD, Michels KB. Genome-wide DNA methylation in neonates exposed to maternal depression, anxiety, or SSRI medication during pregnancy. Epigenetics. 2014;9:964-72. https://doi. org/10.4161/epi.28853.

29. Schroeder JW, Smith AK, Brennan PA, Conneely KN, Kilaru V, Knight BT, et al. DNA methylation in neonates born to women receiving psychiatric care. Epigenetics. 2012;7:409-14. https://doi.org/10.4161/epi.19551.

30. Vangeel EB, Pishva E, Hompes T, van den Hove D, Lambrechts D, Allegaert $\mathrm{K}$, et al. Newborn genome-wide DNA methylation in association with pregnancy anxiety reveals a potential role for GABBR1. Clin Epigenetics. 2017;9:107. https://doi.org/10.1186/s13148-017-0408-5.

31. Viuff AC, Sharp GC, Rai D, Henriksen TB, Pedersen LH, Kyng KJ, et al. Maternal depression during pregnancy and cord blood DNA methylation: findings from the Avon Longitudinal Study of Parents and Children. Trans Psychiatry. 2018;8:244. https://doi.org/10.1038/s41398-018-0286-4.

32. Sharp GC, Salas LA, Monnereau C, Allard C, Yousefi P, Everson TM, et al. Maternal BMl at the start of pregnancy and offspring epigenome-wide DNA methylation: findings from the pregnancy and childhood epigenetics (PACE) consortium. Hum Mol Genet. 2017;26:4067-85.

33. Walden RV, Taylor SC, Hansen NI, Poole WK, Stoll BJ, Abuelo D, et al. Major congenital anomalies place extremely low birth weight infants at higher risk for poor growth and developmental outcomes. Pediatrics. 2007;120:e1512-9.

34. Hollingshead AB. Four factor index of social status. New Haven: Yale University; 1975.

35. Rasmussen KM, Yaktine AK. Weight gain during pregnancy: reexamining the guidelines. Washington DC: National Academy Press; 2009.

36. Kroenke K, Spitzer RL, Williams JB. The PHQ-9: validity of a brief depression severity measure. J Gen Intern Med. 2001;16:606-13.

37. Everson TM, O'Shea TM, Burt A, Hermetz K, Carter BS, Helderman J, et al. Serious neonatal morbidities are associated with differences in DNA methylation among very preterm infants. Clin Epigenetics. 2020;12:1-15. https://doi.org/10.1186/s13148-020-00942-1.

38. Liu J, Siegmund KD. An evaluation of processing methods for humanmethylation450 BeadChip data. BMC Genom. 2016;17:469. https://doi. org/10.1186/s12864-016-2819-7.

39. Aryee MJ, Jaffe AE, Corrada-Bravo H, Ladd-Acosta C, Feinberg AP, Hansen KD, et al. Minfi: a flexible and comprehensive bioconductor package for the analysis of Infinium DNA methylation microarrays. Bioinformatics. 2014:30:1363-9. https://doi.org/10.1093/bioinformatics/btu049.

40. Pidsley R, Zotenko E, Peters TJ, Lawrence MG, Risbridger GP, Molloy P, et al. Critical evaluation of the Illumina MethylationEPIC BeadChip microarray for whole-genome DNA methylation profiling. Genome Biol. 2016;17:208. https://doi.org/10.1186/s13059-016-1066-1.

41. Pidsley R, Wong CC, Volta M, Lunnon K, Mill J, Schalkwyk LC. A data-driven approach to preprocessing Illumina $450 \mathrm{~K}$ methylation array data. BMC Genom. 2013:14:293. https://doi.org/10.1186/1471-2164-14-293.

42. Teschendorff AE, Marabita F, Lechner M, Bartlett T, Tegner J, GomezCabrero D, et al. A beta-mixture quantile normalization method for correcting probe design bias in Illumina Infinium 450 k DNA methylation data. Bioinformatics. 2013;29:189-96. https://doi.org/10.1093/bioinforma tics/bts680.

43. Jaffe AE, Irizarry RA. Accounting for cellular heterogeneity is critical in epigenome-wide association studies. Genome Biol. 2014;15:R31.

44. Zheng SC, Webster AP, Dong D, Feber A, Graham DG, Sullivan R, et al. A novel cell-type deconvolution algorithm reveals substantial contamination by immune cells in saliva, buccal and cervix. Epigenomics. 2018:10:925-40. https://doi.org/10.2217/epi-2018-0037.

45. Everson TM, Marsit CJ, Michael O'Shea T, Burt A, Hermetz K, Carter BS, et al. Epigenome-wide analysis identifies genes and pathways linked to neurobehavioral variation in preterm infants. Sci Rep. 2019;9:1-13.

46. Braun PR, Han S, Hing B, Nagahama Y, Gaul LN, Heinzman JT, et al. Genome-wide DNA methylation comparison between live human brain and peripheral tissues within individuals. Transl Psychiatry. 2019;9:47.
47. Phipson B, Maksimovic J, Oshlack A. missMethyl: an R package for analyzing data from Illumina's HumanMethylation450 platform. Bioinformatics. 2016:32:286-8.

48. MacArthur J, Bowler E, Cerezo M, Gil L, Hall P, Hastings E, et al. The new NHGRI-EBI catalog of published genome-wide association studies (GWAS Catalog). Nucleic Acids Res. 2017;45:D896-901. https://doi.org/ 10.1093/nar/gkw1133.

49. Zhang W, Spector TD, Deloukas P, Bell JT, Engelhardt BE. Predicting genome-wide DNA methylation using methylation marks, genomic position, and DNA regulatory elements. Genome Biol. 2015;16:14. https://doi.org/10.1186/s13059-015-0581-9.

50. Suderman M, Staley JR, French R, Arathimos R, Simpkin A, Tilling K. Dmrff: identifying differentially methylated regions efficiently with power and control. bioRxiv. 2018;508556:1-26.

51. Nemoda Z, Massart R, Suderman M, Hallett M, Li T, Coote M, et al. Maternal depression is associated with DNA methylation changes in cord blood T lymphocytes and adult hippocampi. Transl Psychiatry. 2015;5:e545.

52. Walsh K, McCormack CA, Webster R, Pinto A, Lee S, Feng T, et al. Maternal prenatal stress phenotypes associate with fetal neurodevelopment and birth outcomes. Proc Natl Acad Sci. 2019;116:23996-4005. https:// doi.org/10.1073/pnas.1905890116.

53. Simino J, Sung YJ, Kume R, Schwander K, Rao DC. Gene-alcohol interactions identify several novel blood pressure loci including a promising locus near SLC16A9. Front Genet. 2013;4:277.

54. Zhu Z, Guo Y, Shi H, Liu C-L, Panganiban RA, Chung W, et al. Shared genetic and experimental links between obesity-related traits and asthma subtypes in UK Biobank. J Allergy Clin Immunol. 2020;145:537-49.

55. Domínguez-Cruz MG, Muñoz ML, Totomoch-Serra A, García-Escalante MG, Burgueño J, Valadez-González N, et al. Pilot genome-wide association study identifying novel risk loci for type 2 diabetes in a Maya population. Gene. 2018;677:324-31.

56. Fanous AH, Zhou B, Aggen SH, Bergen SE, Amdur RL, Duan J, et al. Genome-wide association study of clinical dimensions of schizophrenia: polygenic effect on disorganized symptoms. Am J Psychiatry. 2012;169:1309-17.

57. Myung W, Kim J, Lim S-W, Shim S, Won H-H, Kim S, et al. A genomewide association study of antidepressant response in Koreans. Transl Psychiatry. 2015;5:e633.

58. Goes FS, Hamshere ML, Seifuddin F, Pirooznia M, Belmonte-Mahon $\mathrm{P}$, Breuer $\mathrm{R}$, et al. Genome-wide association of mood-incongruent psychotic bipolar disorder. Transl Psychiatry. 2012;2:e180.

59. van der Meer D, Frei O, Kaufmann T, Shadrin AA, Devor A, Smeland OB, et al. Understanding the genetic determinants of the brain with MOSTest. Nat Commun. 2020:11:3512.

60. Davies G, Lam M, Harris SE, Trampush JW, Luciano M, Hill WD, et al. Study of 300,486 individuals identifies 148 independent genetic loci influencing general cognitive function. Nat Commun. 2018;9:2098.

61. Hayashi R, Goto Y, Ikeda R, Yokoyama KK, Yoshida K. CDCA4 Is an E2F transcription factor family-induced nuclear factor that regulates E2Fdependent transcriptional activation and cell proliferation. J Biol Chem. 2006:281:35633-48. https://doi.org/10.1074/jbc.M603800200.

62. Li C, Stoma S, Lotta LA, Warner S, Albrecht E, Allione A, et al. Genomewide association analysis in humans links nucleotide metabolism to leukocyte telomere length. Am J Hum Genet. 2020;106:389-404.

63. Lee JJ, Wedow R, Okbay A, Kong E, Maghzian O, Zacher M, et al. Gene discovery and polygenic prediction from a genome-wide association study of educational attainment in 1.1 million individuals. Nat Genet. 2018:50:1112-21.

64. Eme R. Developmental psychopathology: a primer for clinical pediatrics. World J Psychiatry. 2017;7:159-62.

65. Bas-Hoogendam JM, van Steenbergen H, Tissier RLM, Houwing-Duistermaat JJ, Westenberg PM, van der Wee NJA. Subcortical brain volumes, cortical thickness and cortical surface area in families genetically enriched for social anxiety disorder-a multiplex multigenerational neuroimaging study. EBioMedicine. 2018;36:410-28. https://doi.org/10. 1016/j.ebiom.2018.08.048.

66. Evans GW, Li D, Whipple SS. Cumulative risk and child development. Psychol Bull. 2013;139:1342-96. https://doi.org/10.1037/a0031808. supp. 
67. Burchinal MR, Roberts JE, Hooper S, Zeisel SA. Cumulative risk and early cognitive development: a comparison of statistical risk models. Dev Psychol. 2000;36:793-807.

68. Lawn RB, Anderson EL, Suderman M, Simpkin AJ, Gaunt TR, Teschendorff $A E$, et al. Psychosocial adversity and socioeconomic position during childhood and epigenetic age: analysis of two prospective cohort studies. Hum Mol Genet. 2018;27:1301-8.

69. Lanza ST, Rhoades BL, Greenberg MT, Cox M. Modeling multiple risks during infancy to predict quality of the caregiving environment: contributions of a person-centered approach. Infant Behav Dev. 2011;34:390-406. https://doi.org/10.1016/j.infbeh.2011.02.002.

70. Joubert BR, Håberg SE, Nilsen RM, Wang X, Vollset SE, Murphy SK, et al. 450K epigenome-wide scan identifies differential DNA methylation in newborns related to maternal smoking during pregnancy. Environ Health Perspect. 2012;120:1425-31.

71. Breton CV, Marsit CJ, Faustman E, Nadeau K, Goodrich JM, Dolinoy DC, et al. Small-magnitude effect sizes in epigenetic end points are important in children's environmental health studies: The Children's Environmental Health and Disease Prevention Research Center's Epigenetics Working Group. Environ Health Perspect. 2017;125:511-26.

\section{Publisher's Note}

Springer Nature remains neutral with regard to jurisdictional claims in published maps and institutional affiliations.
Ready to submit your research? Choose BMC and benefit from:

- fast, convenient online submission

- thorough peer review by experienced researchers in your field

- rapid publication on acceptance

- support for research data, including large and complex data types

- gold Open Access which fosters wider collaboration and increased citations

- maximum visibility for your research: over 100M website views per year

At BMC, research is always in progress.

Learn more biomedcentral.com/submissions 